\title{
VECTOR VALUED FUNCTIONS EQUIVALENT TO MEASURABLE FUNCTIONS
}

\author{
J. J. UHL, JR. ${ }^{1}$
}

\begin{abstract}
Let $X$ be a Banach space with dual $X^{*}$ and let $(\Omega, \Sigma, \mu)$ be a finite measure space. Suppose $f: \Omega \rightarrow X$ is weakly measurable. There exists a (norm) measurable $g: \Omega \rightarrow X$ such that $\left\langle x^{*}, f\right\rangle=\left\langle x^{*}, g\right\rangle$ a.e. for each $x^{*} \in X^{*}$ if and only if each set $A$ of positive $\mu$-measure has a subset $B$ of positive $\mu$-measure such that there is a weakly compact convex subset $K$ of $X$ with the property that
\end{abstract}

$$
\left\langle x^{*}, f\right\rangle\left\langle\sup _{x \in K}\left\langle x^{*}, x\right\rangle\right.
$$

$\mu$-almost everywhere on $B$ for each $x^{*} \in X^{*}$.

Throughout this note $(\Omega, \Sigma, \mu)$ is a finite measure space and $X$ is a Banach space with dual $X^{*}$. A function $f: \Omega \rightarrow X$ is weakly measurable if the numerical function $\left\langle x^{*}, f\right\rangle$ is measurable for all $x^{*} \in X^{*}$. A function $f$ : $\Omega \rightarrow X^{*}$ is weak*-measurable if $\langle f, x\rangle$ is measurable for all $x \in X$. A function $g: \Omega \rightarrow X$ is measurable if it is the almost everywhere limit of functions of the form $\sum_{i=1}^{n} x_{i} \chi_{E_{i}}$ where $x_{i} \in X, E_{i} \in \Sigma, \chi_{E}$ is the indicator function of $E$ and $n$ is a positive integer. The object of this note is to characterize those weakly (respectively weak*-) measurable $f: \Omega \rightarrow X$ (respectively $X^{*}$ ) for which there exists a measurable $g: \Omega \rightarrow X$ (respectively $X^{*}$ ) such that $\left\langle x^{*}, f\right\rangle=\left\langle x^{*}, g\right\rangle$ a.e. for each $x^{*} \in X^{*}$ (respectively $\langle f, x\rangle=$ $\langle g, x\rangle$ a.e. for each $x \in X$ ). These characterizations will then be related to a recent theorem of Edgar's [2]. One advantage of the characterizations we are about to prove is that their proofs are direct consequences of Egoroff's theorem, the Dunford-Pettis-Phillips Radon-Nikodým theorem and the separation theorem, familiar tools that have been around for the better part of forty years.

Our theorems will be stated for real Banach spaces only; obvious modifications can be made for complex Banach spaces.

TheOREM 1. Let $f: \Omega \rightarrow X^{*}$ be weak*-measurable. There exists a measurable function $g: \Omega \rightarrow X^{*}$ such that $\langle f, x\rangle=\langle g, x\rangle$ a.e. ${ }^{2}$ for each $x \in X$ if and only if each set $A$ of positive $\mu$-measure has a subset $B$ of positive $\mu$-measure such that there is a weakly compact convex subset $K$ of $X^{*}$ with the property that

Received by the editors March 8, 1977.

AMS (MOS) subject classifications (1970). Primary 28A45.

${ }^{1}$ Supported in part by the National Science Foundation.

${ }^{2}$ The exceptional set is allowed to vary with $x$. 


$$
\langle f, x\rangle\left\langle\sup _{x^{*} \in K}\left\langle x^{*}, x\right\rangle\right.
$$

almost everywhere on $B$ for each $x \in X$.

Proof. Let $f: \Omega \rightarrow X^{*}$ be weakly measurable and suppose there exists a measurable $g: \Omega \rightarrow X^{*}$ such that $\langle f, x\rangle=\langle g, x\rangle$ a.e. for each $x \in X$. Let $A \in \Sigma$ have positive $\mu$-measure. The definition of measurability for $g$ coupled with Egoroff's theorem produces a subset $B$ of $A$ with $B \in \Sigma$ and $\mu(A \backslash B)$ as small as we like such that $g(B)$ is relatively norm compact in $X^{*}$. Let $K$ be the norm closed convex hull of $g(B)$. Then $K$ is weakly compact and evidently $\langle f, x\rangle=\langle g, x\rangle \leqslant \sup _{x^{*} \in K}\left\langle x^{*}, x\right\rangle$ almost everywhere on $B$ for each $x \in X$.

For the converse suppose $f: \Omega \rightarrow X^{*}$ is weak*-measurable. Let $A \in \Sigma$ have positive $\mu$-measure and select $B$ and $K$ as in the hypothesis. Let $x \in X$ and note that both the inequalities

$$
\langle f, x\rangle\left\langle\sup _{x^{*} \in K}\left\langle x^{*}, x\right\rangle \text { and }\langle f,-x\rangle \leqslant \sup _{x^{*} \in K}\left\langle x^{*},-x\right\rangle\right.
$$

obtain almost everywhere on $B$. It follows that $\langle f, x\rangle$ is essentially bounded on $B$. Hence $\int_{B}\langle f, x\rangle d \mu$ exists for all $x \in X$. By a closed graph argument or otherwise [4], this means that for each $E \in \Sigma$, there is an element $F(E \cap B)$ $\in X^{*}$ such that

$$
\langle F(E \cap B), x\rangle=\int_{E \cap B}\langle f, x\rangle d \mu
$$

for all $x \in X$.

Now if for some $E \in \Sigma$ with $\mu(E \cap B)>0$ it happens that $F(E \cap$ $B) / \mu(E \cap B) \notin K$, then view $K$ as a weak ${ }^{*}$-compact convex subset of $X^{*}$, and note that by the separation theorem there is an $x \in X$ such that

$$
\langle F(E \cap B) / \mu(E \cap B), x\rangle>\sup _{x^{*} \in K}\left\langle x^{*}, x\right\rangle \text {. }
$$

But by hypothesis $\langle f, x\rangle \leqslant \sup _{x^{*} \in K}\left\langle x^{*}, x\right\rangle$. Consequently one has

$$
\begin{aligned}
\sup _{x^{*} \in K}\left\langle x^{*}, x\right\rangle & <\langle F(E \cap B) / \mu(E \cap B), x\rangle \\
& =\int_{E \cap B}\langle f, x\rangle d \mu / \mu(E \cap B) \\
& <\int_{E \cap B} \sup _{x^{*} \in K}\left\langle x^{*}, x\right\rangle d \mu / \mu(E \cap B) \\
& =\sup _{x^{*} \in K}\left\langle x^{*}, x\right\rangle,
\end{aligned}
$$

a contradiction which proves $F(E \cap B) / \mu(E \cap B) \in K$ for all $E \in \Sigma$ with $\mu(E \cap B)>0$. An appeal to the Dunford-Pettis-Phillips Radon-Nikodým theorem for the Bochner integral [1], [5] produ'ces a Bochner integrable $g$ : $B \rightarrow K$ such that

$$
F(E \cap B)=\text { Bochner }-\int_{E \cap B} g d \mu
$$

for all $E \in \Sigma$. It follows immediately that 


$$
\int_{E \cap B}\langle f, x\rangle d \mu=\int_{E \cap B}\langle g, x\rangle d \mu
$$

for all $x \in X$ and all $E \in \Sigma$. Thus for each $x \in X$ one has $\langle f, x\rangle=\langle g, x\rangle$ a.e. on $B$. The proof is completed by a standard exhaustion argument [3].

The following corollary is a consequence of the proof of Theorem 1 .

COROllary 2. Let $f: \Omega \rightarrow X$ be weakly measurable. There exists a measurable function $g: \Omega \rightarrow X$ such that $\left\langle x^{*}, f\right\rangle=\left\langle x^{*}, g\right\rangle$ a.e. for each $x^{*} \in X^{*}$ if and only if each set $A$ of positive $\mu$-measure has a subset $B$ of positive $\mu$-measure such that there is a weakly compact convex subset $K$ of $X$ with the property that

$$
\left\langle x^{*}, f\right\rangle \leqslant \sup _{x \in K}\left\langle x^{*}, x\right\rangle
$$

almost everywhere in $B$ for each $x \in X$.

Proof. Suppose $f: \Omega \rightarrow X$ is weakly measurable and there exists a measurable $g: \Omega \rightarrow X$ such that $\left\langle x^{*}, f\right\rangle=\left\langle x^{*}, g\right\rangle$ a.e. for each $x^{*} \in X^{*}$. If $A$ has positive $\mu$-measure, then the Egoroff theorem argument used in the proof of Theorem 1 produces $B \in \Sigma$ and a norm compact convex set $K$ with the desired properties.

The converse is also an evident consequence of a reinterpretation of proof of Theorem 1: Let $f: \Omega \rightarrow X$ be weakly measurable. View $f$ as a weak*measurable function with values in $X^{* *}$. View $K$ as a weakly compact convex set in $X^{* *}$ and note that the proof of Theorem 1 produces a measurable $g$ with values in $K$ such that $\left\langle x^{*}, f\right\rangle=\left\langle x^{*}, g\right\rangle$ a.e. on the set $B$.

Now Theorem 1 and Corollary 2 will be compared to Edgar's theorem. For each $x^{*} \in X^{*}$ and real number $\alpha$, let $\left[x^{*} \leqslant \alpha\right]=\left\{x \in X:\left\langle x^{*}, x\right\rangle \leqslant \alpha\right\}$. For each $x \in X$ and real number $\alpha$, let $[x \leqslant \alpha]=\left\{x^{*} \in X^{*}:\left\langle x^{*}, x\right\rangle \leqslant \alpha\right\}$. To say $f: \Omega \rightarrow X$ is weakly measurable is to say $f^{-1}\left[x^{*} \leqslant \alpha\right] \in \Sigma$ for all $x^{*} \in X^{*}$ and all real $\alpha$. Thus if $W$ is the $\sigma$-field of subsets of $X$ generated by $\left\{\left[x^{*} \leqslant \alpha\right]: x^{*} \in X^{*}, \alpha\right.$ real $\}$, then $f^{-1}(E) \in \Sigma$ for all $E \in W .^{3}$ Similarly if $W^{*}$ is the $\sigma$-field generated by $\{[x \leqslant \alpha]: x \in X, \alpha$ real $\}$, and $f: \Omega \rightarrow X^{*}$ is weak*-measurable, then $f^{-1}(E) \in \Sigma$ for all $E \in W^{*}$.

The following theorem is due to Edgar [2].

COROllaRy 3 (EDgAR). Let $f: \Omega \rightarrow X$ be weakly measurable. If for each $\varepsilon>0$ there is a weakly compact subset $K$ of $X$ such that

$$
\inf \left\{\mu\left(f^{-1}(E)\right): E \in W, E \supseteq K\right\} \geqslant \mu(\Omega)-\varepsilon,
$$

then there is a measurable $g: \Omega \rightarrow X$ such that $\left\langle x^{*}, f\right\rangle=\left\langle x^{*}, g\right\rangle$ a.e. for all $x^{*} \in X^{*}$.

Proof. Let $\varepsilon>0$ and select $K$ as in the hypothesis. There is no loss of generality in assuming that $K$ is convex. Choose $E_{0} \in W$ such that $E_{0} \supseteq K$ and

\footnotetext{
${ }^{3}$ Edgar [2] proves that $W$ is the $\sigma$-field of weak Baire sets.
} 


$$
\mu\left(f^{-1}\left(E_{0}\right)\right)=\inf \left\{\mu\left(f^{-1}(E)\right): E \in W, E \supseteq K\right\} .
$$

It will be shown that if $x^{*} \in X^{*}$, then $\left\langle x^{*}, f\right\rangle \leqslant \sup _{x \in K}\left\langle x^{*}, x\right\rangle$ a.e. on $f^{-1}\left(E_{0}\right)$. Since $\varepsilon>0$ is arbitrary, an appeal to Corollary 2 will complete the proof.

To this end, suppose $x^{*} \in X^{*}$ and $x^{*} f$ is greater than $\sup _{x \in K}\left\langle x^{*}, x\right\rangle$ on a subset of $f^{-1}\left(E_{0}\right)$ of positive $\mu$-measure. Let

$$
E_{1}=E_{0} \cap\left\{y \in X:\left\langle x^{*}, y\right\rangle>\sup _{x \in K}\left\langle x^{*}, x\right\rangle\right\} .
$$

Plainly $E_{1} \in W, E_{1} \cap K=\varnothing, E_{1} \subseteq E_{0}$ and $\mu\left(f^{-1}\left(E_{1}\right)\right)>0$. Thus $E_{0} \backslash E_{1} \supseteq$ $K$ and

$$
\mu\left(\dot{f}^{-1}\left(E_{0} \backslash E_{1}\right)\right)<\mu\left(f^{-1}\left(E_{0}\right)\right),
$$

a contradiction which completes the proof.

Proved similarly is the next corollary.

Corollary 4. Let $f: \Omega \rightarrow X^{*}$ be weak*-measurable. If for each $\varepsilon>0$ there is a weakly compact subset $K$ of $X^{*}$ such that

$$
\inf \left\{\mu\left(f^{-1}(E)\right): E \in W^{*}, E \supseteq K\right\} \geqslant \mu(\Omega)-\varepsilon,
$$

then there exists a measurable $g: \Omega \rightarrow X^{*}$ such that $\langle f, x\rangle=\langle g, x\rangle$ a.e. for each $x \in X$.

The converses of Corollaries 3 and 4 are true. The converse of Corollary 3 is due to Edgar [2] and the converse of Corollary 4 can also be proved by his method. There is a way to make Edgar's proof a bit less abstract than it is by replacing theorems about tight measures on metric spaces by Egoroff's theorem. To illustrate this we shall modify Edgar's proof of the converse of Corollary 3 to prove

THEOREM 5. The converse of Corollary 4 is true.

Proof. Suppose $f: \Omega \rightarrow X^{*}$ is weak ${ }^{*}$-measurable and that there exists a measurable $g: \Omega \rightarrow X^{*}$ such that $\langle f, x\rangle=\langle g, x\rangle$ a.e. for each $x \in X$. Then, for each $\varepsilon>0$, Egoroff's theorem coupled with the definition of measurability produces a set $B \in \Sigma$ with $\mu(\Omega \backslash B)<\varepsilon$ such that $g(B)$ is relatively norm compact. Let $K$ be the closure of $g(B)$. Now since $\langle f, x\rangle=\langle g, x\rangle$ a.e. for each $x \in X$, we see that $\chi_{E} \circ f=\chi_{E} \circ g$ a.e. if $E$ is one of the generators of $W^{*}$. Since the set of $E \subseteq X$ for which $\chi_{E} \circ f=\chi_{E} \circ g$ a.e. is a $\sigma$-field, this equality obtains for all $E \in W^{*}$. Now suppose $E \in W^{*}$ and $E \supseteq K$. Then

$$
\begin{aligned}
\mu\left(f^{-1}(E)\right) & =\int_{\Omega} \chi_{E} \circ f d \mu=\int_{\Omega} \chi_{E} \circ g d \mu \\
& \geqslant \int_{\Omega} \chi_{K} \circ g d \mu \geqslant \mu(B) \geqslant \mu(\Omega)-\varepsilon .
\end{aligned}
$$




\section{REFERENCES}

1. N. Dunford and B. J. Pettis, Linear operations on summable functions, Trans. Amer. Math. Soc. 47 (1940), 323-392.

2. G. A. Edgar, Measurability in a Banach space (preprint).

3. H. B. Maynard, A geometric characterization of Banach spaces having the Radon-Nikodym property, Trans. Amer. Math. Soc. 185 (1973), 493-500.

4. B. J. Pettis, On integration in vector spaces, Trans. Amer. Math. Soc. 44 (1938), 277-304.

5. R. S. Phillips, On linear transformations, Trans. Amer. Math. Soc. 48 (1940), 516-541.

Department of Mathematics, UniverstTy of IllinoIs, Urbana, ILLNois 61801 